Culture et histoire dans l'espace roman

\title{
Les textes hybrides de Sergio Ramírez, entre convention et transgression
}

Nathalie Besse

\section{(2) OpenEdition}

1 Journals

Édition électronique

URL : https://journals.openedition.org/cher/11548

DOI : $10.4000 /$ cher.11548

ISSN : 2803-5992

Éditeur

Presses universitaires de Strasbourg

Édition imprimée

Date de publication : 1 décembre 2012

Pagination : 121-133

ISBN : 978-2-35410-049-0

ISSN : 1968-035X

Référence électronique

Nathalie Besse, "Les textes hybrides de Sergio Ramírez, entre convention et transgression ", reCHERches [En ligne], 9 | 2012, mis en ligne le 14 février 2022, consulté le 16 février 2022. URL : http:// journals.openedition.org/cher/11548; DOI : https://doi.org/10.4000/cher.11548

\section{(c) () (2)}

Ce(tte) œuvre est mise à disposition selon les termes de la Licence Creative Commons Attribution Pas d'Utilisation Commerciale - Partage dans les Mêmes Conditions 4.0 International. 


\title{
Les textes hybrides de Sergio Ramírez, entre convention et transgression
}

\author{
Nathalie BesSe \\ Université de Strasbourg
}

\begin{abstract}
T a littérature se caractérise par l'intertextualité, condition de tout texte Lselon Roland Barthes, ce qui s'avère plus notable encore dans les œuvres modernes et post-modernes. Sergio Ramírez, écrivain de son temps, ne déroge pas à la règle: intergénéricité, métafiction, parodie déterminent également, entre autres aspects, la plupart de ses fictions.

En nous intéressant d'abord à l'intertextualité, nous verrons que les emprunts de Sergio Ramírez révèlent l'empreinte des mythes nationaux nicaraguayens, qu'ils contiennent en filigrane une image abîmée de la nation, comme si dans ses récits à forte inspiration historique, ce romancier autrefois vice-président du gouvernement sandiniste, poursuivait une forme d'action politique, de dénonciation des maux dont souffre son pays. Nous nous pencherons ensuite sur la problématique de l'hybridisme générique, plus particulièrement sur la relation, en l'occurrence bienveillante, entre roman et photos. Nous questionnerons l'aspect transgressif de ces hybridations en nous demandant dans quelle mesure elles subvertissent, ou à tout le moins inquiètent, le genre romanesque, ainsi que l'historiographie officielle et une certaine conception de la vérité. Entre convention et rénovation, ces emprunts, de quelque ordre qu'ils soient, sont souvent porteurs d'une ambivalence et créateurs de tension dans le texte. Pour mieux servir le roman?
\end{abstract}




\section{L'intertextualité et la mort des mythes identitaires nicaraguayens}

Tout texte n'est fait que d'autres textes qu'il intègre et recompose, et se trouve ainsi à la jonction de plusieurs autres. Rien de surprenant dès lors si les romanciers empruntent des fragments d'autres auteurs, ce qui se traduit le plus souvent, dans le cas de Sergio Ramírez, par le recours à la citation. C'est d'ailleurs ainsi que Julia Kristeva qui, en 1966, a forgé le terme d' "intertextualité», en rend compte: «tout texte se construit comme mosaïque de citations, tout texte est absorption et transformation d'un autre texte» (85), ce que ne démentira pas Gérard Genette qui définit quant à lui l'intertextualité comme la «relation de coprésence entre deux ou plusieurs textes, c'est-à-dire, eidétiquement et le plus souvent, par la présence effective d'un texte dans un autre» (8).

Pour Antoine Compagnon, la citation, cet «opérateur trivial d'intertextualité», s'avère en un sensun leurre autant qu'une "force motrice» (44-45), comme si elle possédait le pouvoir de faire progresser le texte. Non qu'elle soit uniquement "dynamique" car le même auteur tient à affirmer par ailleurs la collusion et la confusion en elle de l'actif et du passif, ambiguïté intrinsèque qu'il met en lumière dans la définition qu'il propose de la citation: "un énoncé répété et une énonciation répétante» (46). En ce qui concerne les écrits de Sergio Ramírez, c'est plus l'intergénéricité qui "emporte" le texte, tandis que l'intertextualité concourt à brosser ou à confirmer un contexte socio-historique, une atmosphère, à insister sur des thématiques-phares du roman.

Nous allons voir que ces interférences entre textes ou entre genres révèlent toutes implicitement une certaine appréhension des symboles nicaraguayens: personnages emblématiques ou tristes images d'une nation malmenée par l'Histoire. Dans Margarita, está linda la mar (Prix Alfaguara 1998), le titre de chaque chapitre est un vers de l'inégalable Rubén Darío dont on trouve également quelques strophes dans le corps du roman ce qui se reproduira dans Mil y una muertes qui fera à nouveau la part belle aux frasques du grand poète. Ce qui nous intéresse ici, c'est le sens à accorder à cette intertextualité darienne dans le texte de Sergio Ramírez: il se trouve que Rubén Darío représente l'un des deux paradigmes identitaires du Nicaragua, au côté du rebelle nationaliste Sandino, d'après l'auteur qui estime qu'ils sont au petit pays ce que Cervantes et le Cid sont à l'Espagne (2004b: 198). 
Sandino constitue l'autre emblème national, avons-nous dit; or, au cœur du roman, est interpolée une lettre authentique de Rigoberto López Pérez à sa mère: il s'agit du jeune poète révolté qui tua le dictateur Somoza García en 1956, et qui apparaît comme l'héritier de Sandino. Cette missive qui peut être considérée comme une forme d'intertextualité si l'on voit en elle une longue citation, ou comme un exemple furtif d'intergénéricité puisqu'elle relève du genre épistolaire, rappelle la détermination et la vaillance de ce fils de la nation qui lui sacrifia jadis son existence et compte lui aussi parmi les figures héroïques du Nicaragua.

On observera que les occurrences intertextuelles majeures concernent souvent, comme nous l'avons montré dans des travaux antérieurs, la mort des mythes nationaux qui traverse "à demi-mot" Margarita, está linda la mar. En effet, le grand poète et le jeune rebelle vont être mutilés, celui-ci par les sbires de Somoza qui, sur la demande du dictateur agonisant, lui feront subir une castration post-mortem, celui-là par le docteur Debayle - beau-père du tyran et défenseur des États-Unis pourtant associés à la dépossession et à la barbarie dans l'Histoire du petit pays —, un médecin ambitieux qui précipite malgré lui la mort de Darío avant d'extirper son prodigieux cerveau.

On ajoutera que tout ceci se déroule, comme dans la plupart des romans de Sergio Ramírez, dans un contexte de corruption. Aussi le pouvoir est-il relié au thème excrémentiel dans le prolongement d'une Histoire nationale quant à elle associée aux porcs amenés au Nicaragua par le premier gouverneur: l'impitoyable Pedrarias Dávila. Cette représentation d'un pays amputé et souillé, dont on méprise la souveraineté et la dignité, réapparaît avec la même force dans Sombras nada más (2002) et Mil y una muertes (2004). Les romans de Sergio Ramírez, qui mêlent l'humour au tragique, brocardent à l'envi les représentants du pouvoir. Ainsi Somoza se voit-il ridiculisé dans Margarita, está linda la mar qui, en matière d'intergénéricité, contient un Curriculum vitae satirique non exempt de scatologique, comme si ce texte polymorphe qu'est le roman ne prenait des formes diverses que pour mieux transmettre un même message, affirmer une même condamnation.

Cela dit, ces textes n'épargnent pas non plus Rubén Darío pour lequel le romancier ressent pourtant une profonde admiration et dont il prétend connaître l'œuvre par cœur. Mais préférant offrir l'image, en définitive plus "humaine", d'un poète imparfait plutôt que de le chosifier béatement en un faire-valoir national, il ne rechigne pas à moquer son ivrognerie proverbiale et son irrémédiable neurasthénie. Dans Mil y una muertes, où sont à nouveau narrées, entre autre, les incartades de Darío, quelques vers 
poétiques s'insèrent dans le récit, qui associent l'intertextualité et une ironie chère à Sergio Ramírez, mais pour railler à présent d'autres personnages, plus secondaires. Par exemple, la vierge entre toutes les vierges dont il est question dans la strophe suivante fait allusion à Catalina dont la chasteté n'est pas la première caractéristique puisqu'elle devient la maîtresse du protagoniste, de l'Archiduc Luis Salvador de Majorque et du secrétaire de ce dernier, tous trois finissant par ailleurs dans de cocasses triangles amoureux :

La virgen de las vírgenes es inviolable y pura.

Nadie su casto cuerpo tendrá en la alcoba obscura,

ni beberá en sus labios el grito de victoria,

ni arrancará a su frente las rosas de la gloria... (2004: 249)

Dans ce roman qui relate les péripéties d'un photographe nicaraguayen du XIX ${ }^{\mathrm{e}}$ siècle, Castellón, dont Sergio Ramírez suit la trace en Europe et dont il présente le manuscrit posthume, figurent plusieurs citations empruntées à de grands artistes européens, le plus souvent sous forme d'épigraphes apposées au seuil de chaque chapitre et, chose notable, à nouveau en rapport avec la mort ou plus amplement l'insoutenable fragilité de l'être. Il faut dire que Castellón, le protagoniste, est un photographe amoral et morbide qui cherche à saisir le mystère des corps nus et/ou défunts.

Dans ces fragments intertextuels, il est fait référence à la mort de la façon la plus macabre qui soit par l'allusion au cadavre, seule représentation tangible de la mort pour le vivant. À commencer par le tout premier exergue qui préside au roman : un extrait des Epitafios de Xavier Villaurrutia - dont un vers inspire le titre - qui assimile la mort non plus à la fin de la vie mais au sommeil, tandis que la vie est constituée de plusieurs morts:

Duerme aquí, silencioso e ignorado,

el que en vida vivió mil y una muertes.

Nada quieras saber de mi pasado.

Despertar es morir. ¡No me despiertes!

Mais l'élément qui s’interpénètre avec le récit, est une photo effroyable qui fait de l'intergénéricité un formidable moyen d'amplitude expressive du texte. Et qui contient sans doute à nouveau les démons de Sergio Ramírez.

\section{Roman et photo: intergénéricité et rénovation du genre}

En matière d'intergénéricité, ce sont les photos intercalées dans Mil y una muertes qui retiennent d'abord notre attention, tout particulièrement celle de l'excipit puisqu'à la différence des précédentes qui servent un certain 
réalisme, une certaine vraisemblance, comme autant de subterfuges visant à valider le récit sans rien lui apporter en sus, l'ultime cliché quant à lui semble amplifier le texte, renforce le pathos, pétrifie le lecteur plus que ne l'avait fait auparavant la partie textuelle qui correspond à cette photo sépulcrale. De quoi s'agit-il ? Cette image, qui est censée illustrer un rêve apocalyptique de Castellón, montre le cadavre d'un enfant de trois ans gisant dans la boue et approché par un porc. On apprend, en se rendant sur le site officiel de Sergio Ramírez, que cette photo non retouchée a été prise aux alentours du volcan Casita après que l'ouragan Mitch eut dévasté le pays en 1998.

Comme nous le suggérons dans d'autres études sur Mil y una muertes, il nous semble que cette photo s'inscrit à son tour dans le crépuscule des "dieux" nationaux: nous ne nous attarderons pas sur l'atmosphère mortuaire, ni même sur la fange qui nous paraît prolonger le thème excrémentiel pour mieux dénoncer la corruption ambiante. Nous nous interrogeons en revanche sur cette "mort de l'innocence" dans laquelle nous percevons une possible allégorie de l'Histoire du Nicaragua: ce rêve de mort ne peut-il métaphoriser la mort d'un rêve, en l'occurrence le rêve sandiniste? Il se trouve que Sergio Ramírez intitule le chapitre de son autobiographie consacré au triomphe de la Révolution «La edad de la inocencia» (Ramírez 1999: 57) et que, d'autre part, le rêve prophétique de Castellón le projette dans un Nicaragua des années 1990: or, on sait que la débâcle sandiniste se produisit le 25 février 1990. Si l'on ajoute que le sandinisme avait perdu, selon Sergio Ramírez lui-même ses valeurs éthiques, ce qui est d'après lui le signe d'une mort prématurée (2000: 31 ), il nous semble possible de relier cette mort précoce due à la perte de l'éthique, à l'enfant mort de la photo cerné par la corruption.

Après la mort des mythes nationaux qu'évoque Margarita, está linda la mar, Mil y una muertes ne donne-t-il pas à voir, via l'intergénéricité, la mort de cet autre mythe que fut la Révolution? Une mort par trahison, et une profonde et douloureuse désillusion, dont Sergio Ramírez a souffert plus que d'autres étant donné son engagement et sa foi dans tout le processus révolutionnaire. Cet enfant qui peut figurer une triste allégorie de la Révolution, incarne sans doute également l'Histoire du petit pays agonisant de sa misère et de sa corruption, souillé et oublié de tous. En ce sens, la dernière photo du recueil de contes El reino animal (2006) n'est pas moins intéressante, qui dépeint un enfant des rues sale et à demi nu, pieds et poings liés dans le véhicule dont il a brisé une vitre pour chaparder. Ces enfants sans nom ni visage métaphorisent l'indigence et la détresse du 
Nicaragua, l'injustice sociale aussi, qui mène à la mort, physique dans Mil y una muertes ou symbolique dans El reino animal car enfin quelle existence propre possède le petit vagabond sans identité ni dignité?

Une image dit-elle plus que le texte? est-on en droit de se demander au regard de l'écho persistant que ces clichés éveillent en nous, lecteurs. Sergio Ramírez estime que l'image en elle-même ne libère pas l'imagination autant que le mot:

Existe alguna tendencia contemporánea de agregar fotografías a las novelas, como lo hace el estupendo escritor alemán E. G. Sebald [...]; y las imágenes, que nunca son gratuitas, pasan a ser parte del todo narrativo [...]. Pero lo que en fin de cuentas quiero decir es que los libros siguen funcionando muy bien aun sin fotografías, cuando nos atrapan gracias a sus virtudes narrativas. Las fotografías logran el efecto de crear en el lector un encanto pasajero, pero el artificio no va más allá. Las que se quedan son las palabras (2004c: 67).

Selon l'écrivain, il faut donc des mots pour rehausser l'image, lui conférer une profondeur, sinon un pouvoir suggestif: "ninguna imagen construida fuera de la palabra puede ser un sustituto eficaz, porque la imagen única impide el acto de imaginar que sólo la palabra concede. Allí, en esa infinita variedad de posibilidades, está el reino de la palabra, y su triunfo» (2001: 142). Néanmoins, il admet que la photo a le pouvoir de mettre en évidence certains problèmes graves, l'immédiateté du visuel s'avérant une arme efficace puisqu'elle possède un impact particulier sur les consciences; un impact d'ailleurs différent selon qu'on considère l'image pour elle-même ou dans sa relation à un ensemble, selon qu'elle n'est qu'un ajout ou qu'elle fait partie intégrante d'un tout. Comme l'observe Daniel Grojnowski, " la prégnance du référent est condition nécessaire mais non suffisante des effets propres à l'illustration d'un récit par la photographie. Il faut prendre également en compte... la prégnance de son commentaire!» (176). Dans les différents exemples d'interférences entre représentations verbale et iconique, les images peuvent s'agréger au texte ou s'y intégrer, le "bousculer" ou s'y fondre harmonieusement, tenter un face-à-face ou aspirer à l'interpénétration.

Qu'en est-il dans Mil y una muertes? Dans ce roman agrémenté de photos qui authentifient le texte sans l'altérer, même la dernière image, "consubstantielle" au texte, l'enrichit plus qu'elle ne l'envahit. Chacun étaye l'autre et l'informe, et d'une certaine façon le transcende dans une heureuse complémentarité. Ainsi l'image-choc à laquelle aboutit le roman ne troublet-elle le lecteur que parce que le texte l'a préliminairement investie d'un 
sens: en effet, sans les données textuelles, on peut se demander si le corps de la photo est réellement celui d'un enfant et pas une grande poupée - même s'il est vrai que la taille de ce corps et le ventre gonflé tendent à privilégier la première hypothèse, mais nulle certitude pour autant.

Ce n'est donc pas en elle-même et pour elle-même que cette photo nous heurte mais parce que le texte "l'a fait parler" au préalable, l'a étoffée et lui a donné "vie" au moyen d'une histoire. De ce point de vue, Sergio Ramírez peut estimer qu'en effet l'image seule ne laisse pas libre cours à l'imagination autant que le texte et qu'elle ne saurait prendre le pouvoir dans le roman. Pour autant, n'omettons pas que si c'est indéniablement grâce à l'écriture que la photo finale de Mil y una muertes prend ce relief terrible, c'est elle, et non le texte, que le lecteur garde en lui, comme un malaise entêtant.

Est-ce à dire que l'image peut mettre en péril le texte? Qu'elle le dénature, le dépossède? Que le roman sort affaibli, déformé par l'hybridisme? Sans doute certaines formes d'hybridités tendent-elles à transgresser les genres jusqu'à rendre la notion de genre inopérante, mais d'autres au contraire confirment les catégories génériques (In L'hybride: 14 et 16). Rappelons que tout genre est hybride, ce qui ne revient pas pour autant à considérer que le genre n'existe pas. Et le genre romanesque, peut-être plus que d'autres, se caractérise par une indétermination fondamentale et par la tendance à s'approprier toutes les techniques; on ne peut qu'admettre «la puissance protéiforme du roman et son infinie capacité de renouvellement» (PiégayGros: 18 et 13). Au reste, cette hybridité générique si caractéristique de la littérature moderne et post-moderne, a en réalité toujours coexisté face aux genres établis (In L'hybride: 14).

Mais qu'est-ce qu'un genre qui ne peut être défini, qui n'est pas soumis à «la loi du genre» et qui se développe en s'appropriant les ressorts des autres genres? se demande Nathalie Piégay-Gros qui affirme que le roman est toujours l'autre et, citant Pascal Quignard: «l'autre de tous les genres» (Piégay-Gros: 20 et 18). Cette force d'appropriation et d'assimilation ne rend-elle pas caduque la définition générique? Si définition il y a, car la définition d'un genre littéraire renvoie à une aporie, rappelle Julien Roger, dans la mesure où toute réflexion sur le générique achoppe sur un dilemme manifeste: «apparemment, nous ne pouvons décider de ce qui appartient à un genre, sans savoir déjà ce qui est générique, et pourtant nous ne pouvons savoir ce qui est générique sans reconnaître que tel ou tel élément appartient à un genre» (In L'hybride...: 13). 
Dans tous ses romans, Sergio Ramírez emprunte aux autres genres, de façon plus ou moins subreptice ou déclarée, allusive ou appuyée, mais sans jamais chercher à "nuire" au genre. Il semble qu'il aspire plus à un enrichissement, à une rénovation, pour un renouveau du genre afin que celui-ci ne se sclérose pas dans ses conventions. Ainsi lui arrive-t-il de mêler fugitivement à ses romans des références cinématographiques ou musicales, mais également et surtout des documents de toute sorte qui participent de l'élaboration du récit au point de devenir essentiels sur le plan diégétique: témoignages, déclarations judiciaires, dépêches, lettre d'un personnage de son deuxième roman à l'auteur lui-même (ou plutôt à son double fictionnalisé), rapport révolutionnaire, conversation téléphonique etc. dans Sombras nada más; mail, carte de visite, article de journal dans El cielo llora por mí.

Tous ces documents, et plus encore les photos de Mil y una muertes et de El reino animal, créent un effet de réel pour parler comme Roland Barthes, défictionnalisent le récit précisément pour mieux favoriser l'immersion fictionnelle. Dans ces romans de tournure réaliste le plus souvent, les documents certifient la véracité du récit comme l'explique Sergio Ramírez:

Manuscritos, cartas, telegramas, expedientes judiciales, viejos periódicos, crónicas olvidadas, traducciones; en esa suma de apócrifos - aunque la palabra apócrifo la demos aquí por prohibida, ya que niega o descubre el mecanismo de la invención - encontramos las piezas prefabricadas que solas, $o$ al ensamblarse, pasan a formar parte del cuerpo narrativo, $o$ a veces a sustituirlo por completo. Son formas del haber estado allí. Haber estado allí es, también, demostrar la posesión de los materiales narrativos; ser el dueño del secreto, y estar dispuesto a revelarlo (2001: 59).

Cela étant, et comme il le précise lui-même, il s'agit de textes apocryphes qui en outre vont être mis en question dans le roman, au point d'invalider parfois ce dernier. L'intertextualité et l'intergénéricité auxquelles recourent volontiers les romans de Sergio Ramírez doivent donc composer, sinon se débattre, avec une force adverse: le texte se questionnant lui-même. Ce qu'elles avancent, il le met en question; ce qu'elles attestent, il l'ébranle lorsque dans les derniers chapitres il se retourne sur lui-même et met en doute la crédibilité du narrateur ou la véridicité des faits narrés jusque là. L'intertextualité et l'intergénéricité se retrouvent donc au cœur d'une ambivalence textuelle, parties prenantes d'une tension entre réalisme et improbable vérité. 


\section{La théâtralisation de l'Histoire: intergénéricité et transgression}

Les documents fictionnels abondent dans les romans de Sergio Ramírez; il s'agit même parfois de chapitres entiers attribués à tel ou tel écrivain de renom comme les deux chapitres d'ouverture des deux grandes parties de Mil y una muertes dont l'un aurait été rédigé par Rubén Darío. Forte présence du texte apocryphe donc, c'est-à-dire de la fiction dans la fiction, à quoi s'ajoute l'insistance sur le droit d'inventer et de ne pas dire la vérité: ainsi en est-il dans Sombras nada más où le romancier avoue avoir laissé sans correction l'erreur d'un des personnages "por razones de novelista", dernière ligne de l'ouvrage; même ambiguïté dans Mil y una muertes dont le final rend plus que douteux le manuscrit de Castellón qui constitue pourtant la base même du roman.

L'intertextualité et l'intergénéricité ont beau favoriser la vraisemblance, les dernières lignes du roman frappent de soupçon tout l'ouvrage jusqu'à son essence même: l'autoréférentialité du récit signe sa mise en accusation pour ainsi dire, et pas seulement celle de tel ou tel roman mais plus amplement celle de tout roman en lui-même, impropre à délivrer la vérité - et n'ayant pas vocation à le faire? -, mais apte à inventer, captiver et leurrer l'"hypocrite" lecteur qui feindra volontiers la crédulité. Sergio Ramírez s'inscrit en cela dans le courant de pensée moderne et post-moderne qui, après la mort des utopies et des grands crédos, se défie de la quête de la vérité et pense que la littérature, comme l'Histoire et quelque science humaine que ce soit, ne peut offrir qu'une vérité, subjective et parcellaire.

Romans qui se déconstruisent eux-mêmes, appréhension fragmentaire d'une vérité qui serait plurielle, cela n'empêche pas que le roman délivre, comme le mythe et tout récit fictif ou "symbolique", une vérité de l'homme, peut-être plus profonde, plus universelle que celle que prétendent rendre les faits. Si donc la métafiction se charge de saborder les bonnes œuvres de l'intertextualité et de l'intergénéricité en rappelant que ce qui avait l'air réaliste n'est sans doute pas réel ou vrai, le roman n'en perd pas pour autant sa "capacité" de réflexion sur l'homme et sa réalité d'homme, sociale, historique, existentielle: les citations et les photos servent cette approche "humaine".

D'autre part, le narrateur non digne de confiance s'avère plus intéressant, d'après Paul Ricœur, du point de vue de l'appel à la liberté et à la responsabilité du narrataire: un lecteur désorienté n'est-il pas davantage appelé à réfléchir, demande le philosophe (236-237): 
Le narrateur non digne de confiance dérègle ces attentes, en laissant le lecteur dans l'incertitude sur le point de savoir où il veut finalement en venir. Ainsi le roman moderne exercera-t-il d'autant mieux sa fonction de critique de la morale conventionnelle, éventuellement sa fonction de provocation et d'insulte, que le narrateur sera plus suspect et l'auteur plus effacé [...] (237).

Cette contestation des conventions souligne l'aspect transgressif du roman moderne. Or, à bien y regarder, l'hybridisme suppose également une transgression de l'ordre ancien, un refus de rester dans la "mêmeté », dans le "pur» (In L’hybride: 12). L’intergénéricité qui, dans les romans de Sergio Ramírez, sert un effet de réel et se trouve a priori du côté de la convention "réaliste", présente donc elle aussi en définitive sa part de transgression et de subversion. Si elle authentifie les faits narrés dans le roman et possède par là une fonction "conservatrice", elle déjoue la rigidité du genre, ou joue avec sa malléabilité.

On retrouve cette ambivalence de l'intergénéricité dans les romans de Sergio Ramírez avec les nombreux exemples de bribes théâtrales intégrées au récit. Cette théâtralisation de l'action qui semble associer le roman à une "comédie", reflète plutôt la grande comédie humaine et finalement une sorte de theatrum mundi. La parodie chère à Sergio Ramírez et aux écrivains de son temps, traverse ces fragments théâtraux, et fait souffler à son tour un vent de transgression dans le roman, outre la "mise en scène" assortie de doubles aux surnoms empruntés au cinéma et de déguisements qui sousentendent une carnavalisation - et donc une grotesquisation? - des faits.

Le recours au texte théâtral contribue, dans Margarita, está linda la mar, à la fictionnalisation de l'Histoire et ébranle l'historiographie, ce qui représente à nouveau une forme évidente de subversion. Divers extraits courts (d'une à quatre pages), assortis de didascalies, émaillent le roman et ont à voir, pour la plupart, avec le fait majeur du récit, à savoir un complot contre le dictateur: ainsi “assiste"-t-on - puisque le texte théâtral semble "présentifier" personnages et faits - à l'élaboration de cette cabale dans le café la Casa Prío de León (154-161); cette action décisive pour le pays est rattachée à un destin, une Volonté supérieure marquée par le passage de la planète Mars au plus près de la Terrequi favorise l'éviction d'un Somoza antéchristique (217-221); on peut également lire la conversation entre Somoza et le docteur Debayle, celui-là demandant en mariage la fille de celui-ci dans la crypte où gît le grand poète Darío au beau milieu du bruit des maçons (317-319); ou encore ces quelques lignes entre un Somoza agonisant 
à l'hôpital et l'un de ses colonels auquel il demande qu'on castre Rigoberto López Pérez pour lui faire une «sopa de huevos» (348).

D’autres exemples de "théâtralisation" du texte composent ce roman: un Intermezzo tropical au cœur du récit comprenant le curriculum vitae peu orthodoxe du dictateur et la lettre de Rigoberto à sa mère avant son coup d'éclat (163-178); la répétition au théâtre Darío d'une pièce dans laquelle Rigoberto a une réplique: "buenas noches, padrecito", et en parallèle, le théâtre González où se tiendra la cérémonie durant laquelle le même jeune rebelle tirera sur le "père de la nation" (226); adresses du narrateur au lecteur, apartés, plongée sur la Place où "se joue" l'Histoire du pays comme si narrateur et lecteur se partageaient une loge en surplomb.

On le voit, ces accointances du roman avec le théâtre ne sont pas dénuées d'une résonance historique puisqu'elles concernent un fait déterminant de l'Histoire du pays. Cette dernière, comme le roman, apparaît d'ailleurs, du fait même de la théâtralisation, à la fois comme un jeu à ne pas prendre au sérieux, et comme une sorte de comédie humaine, c'est-à-dire au contraire une histoire universelle dont on a beaucoup à apprendre: entre dérision ou leçon de l'Histoire, l'intergénéricité constituée par le théâtre, semble à nouveau ambivalente, au carrefour de sens contradictoires.

Sombras nada más propose la même spectacularisation de l'Histoire: à quelques jours du triomphe sandiniste, les jeunes révolutionnaires capturent un ancien bras droit du dictateur qu'ils soumettent à un tribunal populaire. Or, c'est moins la vérité que la capacité du prisonnier à séduire les auditeurs qui lui vaudra des applaudissements libérateurs ou un irrévocable «Paredón!». Déjà dans Castigo Divino (1988), la mise en scène d'un procès théâtralisait l'événement, déjà la vérité échappait dans l'imbroglio de versions discordantes et de mystères non élucidés, déjà la parodie traversait la relecture de l'Histoire et contribuait, avec le mélange des discours juridique, policier, historique, journalistique, feuilletonesque, à démonter l'hypocrisie du discours dominant, l'interdiscursivité servant autant la subversion de chaque type de discours qu'une critique sociale.

Mais dans le roman policier El cielo llora por mí (2008), la vérité ne se dérobe pas indéfiniment: l'enquête, en dépit de ses méandres trompeurs, ainsi que les valeurs éthiques de la révolution sandiniste - quoique celleci ait démérité et reste associée aujourd'hui pour beaucoup de nicas à une trahison - permettent d'appréhender finalement les malfaiteurs, de démanteler pour un temps un puissant réseau de narcotraficants et de dévoiler bien sûr la corruption au plus haut niveau des différentes instances 
représentant l'autorité. Décomposition morale versus éthique, un terme fondamental pour Sergio Ramírez, qui rend le combat possible, la justice plausible: encore faut-il ne pas se taire, encore faut-il dire et montrer, fûtce au moyen de la fiction, pour que le roman, tout en préservant l'art du bien écrire et le souci de captiver le lecteur au moyen d'une intrigue de qualité, permette également, comme l'a toujours souhaité cet écrivain actif, la participation à ce monde.

Dans la plupart des fictions de Sergio Ramírez, l'intertextualité participe d'une toile de fond et d'un message - ici le déclin des grands mythes nationaux et l'agonie d'un Nicaragua corrompu -, tandis que l'intergénéricité bien souvent s'allie à l'action, se trouve au cœur même de la diégèse. Dans le cas très particulier des photos, nous avons pu voir qu'une complicité s'établit entre le roman et l'image, que le texte donne du sens au cliché qui lui confère à son tour un volume.

Les différentes occurrences d'hybridité génériques visent un effet de réel, et sont donc du côté du réalisme pour ainsi dire, mais les documents apocryphes, la légitimation de l'invention romanesque, et une métafiction qui questionne l'ensemble du récit, nuisent sciemment à sa crédibilité. Concernant cette subversion des conventions, nous avons rappelé qu'une forme de transgression définit l'hybridisme en soi, même s'il est vrai que Sergio Ramírez ne cherche aucunement à porter préjudice au genre lorsqu'il y recourt; de même la théâtralisation, et avec elle une certaine carnavalisation et une évidente parodie de l'Histoire, une remise en cause de l'idée même de vérité, et une autoréférentialité destructive, concourent délibérément à troubler la confiance du lecteur.

Les conventions réalistes ne musellent donc pas les aspirations transgressives; mais cette transgression, loin de saborder le roman, opère dans le sens d'une rénovation salutaire du genre, permettant des fictions qui impliquent davantage le lecteur, plus surprenantes, semblables à l'existence où la vérité échappe toujours.

\section{Bibliographie}

Besse N., 2008, «Satire et mort des mythes nationaux dans Margarita, está linda la mar de Sergio Ramírez", in La satire en Amérique latine, formes et fonctions, volume 2, «La satire contemporaine». América - Cahiers du CRICCAL, $\mathrm{n}^{\circ} 38$, Presses de la Sorbonne Nouvelle - Paris III, p. 19-25. 
Besse N., «La muerte en Mil y una muertes ¿y las ilusiones perdidas de Sergio Ramírez?». Página oficial de Sergio Ramírez, sección "Crítica»: http://www. sergioramirez.org.ni/indexcritica.html

Compagnon A., 1979, La seconde main ou le travail de la citation, Seuil, Paris.

Genette G., 1982, Palimpsestes. La littérature au second degré, Seuil, Paris.

Grojnowski D., 2005, "Le roman illustré par la photographie», in Texte/Image: nouveaux problèmes, Colloque de Cerisy, Presses Universitaires de Rennes, p. 171-184.

Kristeva J., 1969, Semeiotikè, Seuil, Paris.

L'hybride/Lo híbrido, 2005, in Cultures et Littératures hispano-américaines, Les Ateliers du SAL, Indigo et Côté-Femmes Éditions, Paris.

Piégay-Gros N., 2005, Le roman, Collection Corpus, Flammarion, Paris.

Rabau S., 2002, L'intertextualité, Collection Corpus, Flammarion, Paris.

Ramírez S., 1988, Castigo Divino, Mondadori, Madrid.

Ramírez S., 1998, Margarita, está linda la mar, Ediciones Alfaguara, Madrid.

Ramírez S., 1999, Adiós Muchachos. Memoria de la revolución sandinista, El País/ Aguilar, Madrid, México.

Ramírez S., 2000, Oficios Compartidos/Un sandinismo en el que creer, CRLAArchivos, "Conferencias en el centro", Poitiers.

Ramírez S., 2001, Mentiras verdaderas, Alfaguara, México.

Ramírez S., 2002, Sombras nada más, Alfaguara, México.

Ramírez S., 2004a, Mil y una muertes, Alfaguara, México.

Ramírez S., 2004b, Una vida por la palabra, Entrevista de Silvia Cherem con Sergio Ramírez, Prólogo de Carlos Fuentes, Fondo de Cultura Económica, México.

Ramírez S., 2004c, El viejo arte de mentir, Cuadernos de la Cátedra Alfonso Reyes, Fondo de Cultura Económica, México.

Ramírez S., 2006, El reino animal, Alfaguara, Madrid.

Ramírez S., 2008, El cielo llora por mí, Alfaguara, México.

Ramírez S., página oficial: http://www.sergioramirez.org.ni/

RamírezS., blogElBoomeran (g): http://www.elboomeran.com/blog/7/sergio-ramirez/ Ricoeur P., 1985, Temps et récit III. Le temps raconté, Seuil, Paris. 